rates were $88 \%$ and $100 \%$, respectively. The median serum creatinine level was $106 \mu \mathrm{mol} / \mathrm{I}$ at 12 months. Only one episode of acute rejection occurred, corresponding to a rate of $13 \%$. No cases of new-onset diabetes mellitus, cancer or serious infection were reported, and viral load consistently remained within the boundaries specified by the inclusion criteria.

Original article Gruber SA et al. (2008) Preliminary experience with renal transplantation in HIV+ recipients: low acute rejection and infection rates. Transplantation 86: 269-274

\section{Sirolimus reduces left ventricular hypertrophy in renal transplant recipients}

Persistent left ventricular hypertrophy (LVH) after renal transplantation is associated with increased long-term mortality, and immunosuppressants might have a role in the development of this complication. Accordingly, Paoletti et al. examined changes in LVH in 13 deceased-donor renal transplant recipients who were switched from calcineurin inhibitors to sirolimus after developing biopsy-proven calcineurin-inhibitor nephrotoxicity or chronic allograft nephropathy.

Echocardiographic changes over 12 months of therapy were compared with those in a control group composed of 26 age-matched patients who had received a single renal allograft during the same year as the study population but who remained on calcineurin inhibitors. Blood pressure of $130 / 80 \mathrm{mmHg}$ or less was targeted by use of antihypertensives that do not act via the renin-angiotensin system.

In the sirolimus group, 12 (92\%) patients achieved a decrease in left ventricular mass index (i.e. regression of LVH), whereas 10 (38\%) patients in the control group achieved such a decrease $(P=0.002)$. The average decrease in left ventricular mass index was $8.6 \pm 2.4 \mathrm{~g} / \mathrm{m}^{2.7}$ greater in the sirolimus group than in the control group $(P<0.001)$ and was largely attributable to decreased left ventricular wall thickness.

The one patient in the sirolimus group who did not display LVH regression exhibited a marked worsening of blood pressure control, confirming the contribution of hypertension to LVH. Overall, however, decreases in blood pressure occurred in both the study cohort and the controls and were not significantly different between the two groups, indicating that nonhemodynamic mechanisms might also affect LVH.

Original article Paoletti E et al. (2008) Effect of sirolimus on left ventricular hypertrophy in kidney transplant recipients: a 1-year nonrandomized controlled trial. Am J Kidney Dis 52: 324-330

\section{Is it better to perform kidney transplantation during the day than at night?}

A recent study published in Transplantation Proceedings suggests that kidney transplantations performed during the night are at greater risk of complications and graft failure than those performed during the day, possibly because the surgeon is less alert at night.

Fechner and colleagues analyzed data from 260 deceased-donor kidney transplantations performed at a single German center during 1994-2004; the transplantations were divided into 'day' (initiated between $0800 \mathrm{~h}$ and $2000 \mathrm{~h}$; $n=166$ ) and 'night' (initiated between $2000 \mathrm{~h}$ and $0800 \mathrm{~h} ; n=94$ ) procedures. Patients received basiliximab induction, and ciclosporin, mycophenolate mofetil and prednisone as maintenance therapy.

Overall graft failure rate was $8.1 \%$ during the first year and $12.7 \%$ at 5 years after transplantation. Logrank testing revealed that operations initiated at night were at significantly higher risk of long-term graft failure than those initiated during the day $(P<0.05)$. In total, 9.1\% of patients required reoperation for surgical complications within 30 days of transplantation; reoperation was associated with an increased risk of graft failure $(P<0.05)$. Night-time operations were associated with a higher incidence of surgical complications (hence reoperation) than daytime operations $(16.8 \%$ vs $6.4 \% ; P<0.01)$. A cold ischemia time $>18 \mathrm{~h}$ did not significantly increase the risk of graft failure.

The authors conclude that although delaying transplantation of a 'night kidney' to the following day will prolong cold ischemia time, such a delay might be worthwhile in terms of the reduced risk of complications and graft failure associated with daytime transplantation.

Original article Fechner Get al. (2008) Kidney's nightshift, kidney's nightmare? Comparison of daylight and nighttime kidney transplantation: impact on complications and graft survival. Transplant Proc 40: 1341-1344 